$\mathbb{P}$ periodica polytechnica

Chemical Engineering

$51 / 2(2007) 97-12$

doi: 10.3311/pp.ch.2007-2.02

web: http://www.pp.bme.hu/ch

(c) Periodica Polytechnica 2007

RESEARCH ARTICLE

\section{Investigation of evolved layer structure of Ba-containing emission materials}

\author{
Ildikó Beck / Katalin V. Josepovits / János Sneider
}

Received 2006-10-03

\section{Abstract}

The emission material in High Intensity Discharge (HID) lamps plays a significant role in lowering the electrode work function and thus the lamps' operating temperature. Bacontaining rare earth- and alkali earth tungstate materials are commonly used as cathode thermionic emission materials because of the production of high intensity discharge.

The goal of this present work is to model a cathode tip surface evolved during sintering and to compare the physical and chemical properties of emission materials currently used in the industry. In order to achieve this goal, we investigated and compared the layer structure of $\mathrm{Ba}-\mathrm{Ca}$ - and $\mathrm{Ba}-\mathrm{Y}$-containing emission materials evolved on polycrystalline tungsten foils. Simultaneously, the tendency of the work function was also monitored as a function of $\mathrm{Ba} / \mathrm{BaO}$ layer thickness. The $\mathrm{Ba}$ coverage of cathode is one of the most important factors during the lifetime of HID lamps. The initial Ba diffusion was also examined. We also proposed a layer model, valuable for the structures occurring during the operation of lamps.

The chemical composition of the flat samples was analysed by $X$-ray Photoelectron Spectroscopy (XPS) and the electron emission properties by Work Function Spectroscopy (WFS).

\section{Keywords}

discharge lamps · Work Function Spectroscopy · X-Ray Photoelectron Spectroscopy · emission material

\section{Ildikó Beck}

Department of Atomic Physics, Surface Physics Laboratory, BME, H-1111, Budapest, Budafoki út 8., Hungary

e-mail: ildiko.beck@ge.com

\section{Katalin V. Josepovits}

Department of Atomic Physics, Surface Physics Laboratory, BME, H-1111, Budapest, Budafoki út 8., Hungary

e-mail: flip@eik.bme.hu

\section{János Sneider}

General Electric Hungary Inc. Consumer \& Industrial, H-1340 Budapest XIII., Váci út 73-77., Hungary

e-mail: janos.sneider@ge.com

\section{Introduction}

Barium has a considerable importance in lighting technology as a work function lowering element, which is shown by the Richardson-Dushmann equation [1], where a lower work function and a higher thermionic current are achieved at the same temperature. For that reason, the investigation of the work function is critical to these lamps. In the commercial high-pressure discharge lamps the mixture of Ba-containing rare earth- and alkali earth tungstate materials are used as an emission material to lower the electrode work function [2]. The emission material that coats the tungsten electrode coils vaporizes by a dissociative process at high operating temperature $\left(1600^{\circ} \mathrm{C}\right)$, generating free $\mathrm{Ba}$ at the surface, as a result of the reaction between the polycrystalline tungsten cathode and the emission material [3]. The Ba coverage results in an improved lamp performance by lowering the electron work function $(\Phi)$ of the tungsten electrodes, and consequently by lowering the operating temperature $(\Phi g g=4,5 \mathrm{eV}$ for clean $\mathrm{W}$ surface [4], $\Phi=2,0-2,30 \mathrm{eV}$ for $\mathrm{BaO}$ monolayer on $\mathrm{W}$ at $1000-1200 \mathrm{~K}[5-7])$.

Many efforts have been taken to investigate and to understand the mechanism of thermionic cathodes during their operation time and it is also ascertained that either $\mathrm{Ba}$ or $\mathrm{BaO}$ layers can decrease the work function of the cathode. In 1957 Rittner et al. [4] found the following three facts: (i) the emitting surface of the cathode consists not only of barium on clean tungsten, (ii) oxygen is an important constituent of the dipole layer throughout the cathode life, and (iii) the oxygen coverage appears to be nearly monoatomic. Based on experimental results, Forman [5] suggested that a barium multilayer is unstable at the operating temperature of cathodes. Thus, in the initial stages of lifetime the cathode behaves as a slightly oxidized tungsten surface covered by a barium monolayer at normal operating temperatures. If the arrival rate of barium to the surface is slow - it may occur if the pores of tungsten are depleted of barium during aging - only a partial monolayer of barium can be sustained in thermodynamic equilibrium on the surface. Rittner has found a Ba monolayer on the surface over the entire cathode life strongly bounded to the tungsten by an underlying nearly complete monolayer of oxygen [8]. Rittner also observed that the end of lamp life is in 
connection with the barium exhaustion from the porous tungsten matrix. The results of Haas and Springer [9] establish the presence of a partial monolayer of barium oxide on the surface, while Jones [10] has experimental evidence of an equilibrium balance between barium and barium oxide.

Taking into account that the partial monolayer coverage leads to the lowest work function and its main constituents are Ba and $\mathrm{O}$, since the seventies it has been an accepted fact that this is the preferred state of the cathode surface [6,7, 11, 12].

Our goal was to model a cathode tip surface evolved during sintering, and we proposed a layer model valuable for the structures occurring during the operation of lamps. To achieve this goal, we carried out model experiments on Ba covered flat tungsten plates, prepared under industrial conditions in order to model a cathode tip surface. We simultaneously investigated the tendency of the work function and the chemical composition at room temperature, under oxygen-free UHV atmosphere, as a function of $\mathrm{Ba} / \mathrm{BaO}$ layer thickness.

\section{Experimental}

The experiments were carried out at room temperature in a combined surface analytical system allowing for electron excited WFS (Work Function Spectroscopy) and for XPS (X-ray Photoelectron Spectroscopy) in the same UHV chamber with a background pressure below $10^{-6} \mathrm{~Pa}$, pumped with an ion getter pump. The WFS measurements were performed using a VG Microtech LEG200 electron gun, with a minimum spot diameter of $200 \mathrm{~nm}$. Primary electrons were accelerated by $0.25-$ $9 \mathrm{keV}$. A VG Microtech CLAM2 truncated hemispherical analyser with an energy resolution of $0,3 \mathrm{eV}$ was used for electron energy analysis.

The WFS method uses the low kinetic energy region $(0-30$ $\mathrm{eV}$ ) of the secondary electron energy spectrum [13,14]. The electrons with the lowest energy but capable of passing the surface potential barrier of the sample provide the onset of the kinetic energy spectrum. The onset of the energy spectrum is used to determine the work function $(\Phi)$ value and its shift indicates the change of work function. In this present work the tangent method was used to obtain $\Phi$ (for details, see [15]).

For the XPS investigation an $\mathrm{Mg} \mathrm{K} \alpha \mathrm{X}$-ray source was used. In order to achieve a different $\mathrm{Ba} / \mathrm{BaO}$ layer thickness, the surface was cleaned in situ by a differentially pumped argon ion gun. The applied current density was $10^{-6} \mathrm{~A} / \mathrm{cm}^{2}$, resulting in a sputtering rate of $0,2 \mathrm{~nm} / \mathrm{min}$ on the $\mathrm{SiO}_{2}$ plate. The applied ion energy was $3 \mathrm{keV}$.

In this work we modelled the interaction between the emission material and the tungsten surface by carrying out experiments on tungsten foils. The model samples were polycrystalline $\mathrm{W}$ foils (average grain diameter of $1 \mu \mathrm{m}$ ) with a hollow (diameter of $0,2 \mathrm{~mm}$ ) in their centres. A Ba coating was obtained on top of tungsten, applying the conventional industrial technology. The Ba-containing compound was deposited onto one side of the $\mathrm{W}$ plate in the form of an alcohol-containing suspension.
After sintering in argon compositional changes occurred at elevated temperature, enabling the Ba to diffuse through the hole to the opposite, clean surface. This surface was used to record the XPS spectra and it was also used to monitor the work function of Ba layers as a function of layer thickness.

By resistive heating of the sample holder and thus the sample in UHV conditions, it was possible to explore the temperature dependence of diffused $\mathrm{Ba}$ amount on tungsten surface. In the next step of the investigation the tungsten foils were heated in several steps up to $700^{\circ} \mathrm{C}$, and they were held for $6 \mathrm{~min}$ at each elevated temperature. As a result, the $\mathrm{Ba}$ diffused through the hole to the tungsten surface from the source of the emission material. Due to the UHV environment, the transportation could take place exclusively via diffusion. The Ba3d and W4f photoelectron peaks and work function values were recorded as a function of temperature. The temperature was measured by an optical pyrometer through the window of the chamber.

For the experiments we used Ba-Ca-containing and $\mathrm{Ba}-\mathrm{Y}$ containing emission materials.

\section{Results and Discussion}

After the sintering process of the tungsten foils, we detected the XPS spectra of $\mathrm{Ba}_{3} \mathrm{~d}_{5 / 2}$, appearing at $780 \mathrm{eV}$ to establish its chemical bounding. The Gauss type shape as well as the binding energy value indicated that $\mathrm{BaO}$ layer evolved on the tungsten surface [16]. Looking at the XPS spectrum of W4f peak, two bonding states could be at once distinguished: elemental and oxidized $\mathrm{W}$. The doublet of the elemental $\mathrm{W}$ appears at $30,5 \mathrm{eV} / 32,65 \mathrm{eV}$, and that of the oxidized $\mathrm{W}$ appears at 35,1 $\mathrm{eV} / 37,25 \mathrm{eV}$. The $\Phi$ work function was measured at $4,1 \mathrm{eV}$ in case of both emission materials. This value is lower than that of the cleaned and oxidized $\mathrm{W}$ surface $(4,5 \mathrm{eV}$ for clean $\mathrm{W}$ surface and 5,67 eV for oxidized $\mathrm{W}$ surface, respectively) and higher than it is suggested for the $\mathrm{BaO}$ covered tungsten surface $(2,0$ $2,30 \mathrm{eV})$. This observation could be connected to the oxygen adsorption to the surface: from the literature it is well known that the adsorbed oxygen increases the work function at least by $2 \mathrm{eV}$ [17-[19].

In the first part of the experiment we investigated the tungsten surface after sintering process. The depth profile was achieved by $\mathrm{Ar}^{+}$ion bombardment and the tendency of the work function and XPS spectra of Ba3d, O1s, W4f, Ca2p and C1s were recorded, as a function of $\mathrm{BaO}$ thickness. In Fig. 1 the XPS concentration and work function values are shown as a function of the sputtering time in case of $\mathrm{Ba}-\mathrm{Ca}$-containing emission material. In Fig. 1 the first data points show the chemical composition after sintering. The oxygen suppresses the effect of barium, and the work function is influenced mainly by oxygen, resulting in a higher work function value, as it is discussed above. After 3 min of ion bombardment the amounts of the physisorbed oxygen and carbon as well as barium were decreasing, leading to a decreasing work function. After 6 min sputtering time the concentration of $\mathrm{C}$ and $\mathrm{Ca}$ was below the XPS detection limit (1\%) 


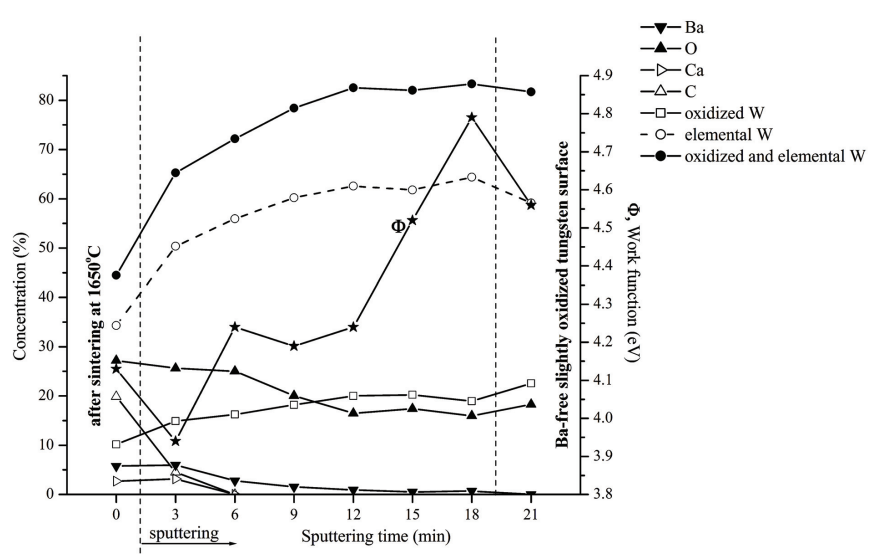

Fig. 1. Work function and XPS concentration of Ba3d, O1s, Ca2p, W4f and $\mathrm{C} 1 \mathrm{~s}$ as a function of the sputtering time in case of Ba-Ca-containing emission material

and, in the meantime the amount of $\mathrm{Ba}$ also decreased, resulting in an increasing work function. By 12 min sputtering time the most part of Ba left the surface. This led us to the conclusion that the entire not-tungsten-bound $\mathrm{O}$ also disappeared. In the meantime the concentration of the elemental and oxidized tungsten increased as a result of the clearing surface. The oxygen had a constant ratio (20\%), so it is most probably bounded chemically to the tungsten. After 15 min of sputtering time the amount of $\mathrm{O}$ did not change while the $\mathrm{Ba}$ was continuously eliminated from the surface, approaching to a Ba-free $\mathrm{W}$ foil. At the same time, the work function drastically increased. This is a proof that even a small amount of $\mathrm{Ba}$ is able to affect the work function. After 21 min sputtering the surface corresponds to a Ba-free and slightly oxidized tungsten foil, and the measured work function value $(\Phi=4,6 \mathrm{eV})$ is in a good agreement with the value known from the literature.

The changes to the chemical composition by sputtering were also traced for the Ba-Y-containing emission material. Fig. 2 shows the XPS concentration and work function values as a function of the sputtering time.

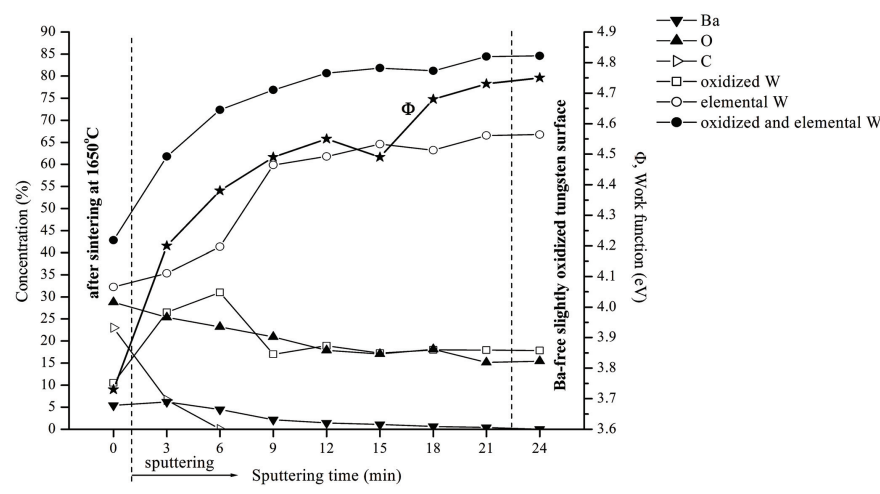

Fig. 2. Work function and XPS concentration of Ba3d, O1s, W4f and $\mathrm{C} 1 \mathrm{~s}$ as a function of the sputtering time in case of $\mathrm{Ba}$-Y-containing emission material

The Y3d could not been detected on the surface of this sample; it probably sintered into the $\mathrm{W}$ during the sample preparation. Similarly to Fig. 1. there was no significant change in

\begin{tabular}{|c|}
\hline Adsorbed O and C \\
\hline Ba-O layer \\
\hline Tungsten oxide and elemental W $($ Ba-YWox/Wel $>$ Ba-CaWox/Wel) \\
\hline Elemental W \\
\hline
\end{tabular}

Fig. 3. The proposed layer structure that evolves during sintering on the cathode tips surface

the amount of Ba after 3 min of sputtering time. By 6 min ion bombardment, the concentration of the physisorbed carbon decreased below the detection limit. Despite the expected decreasing work function it had an increasing trend after eliminating the surface contaminants. By comparing the results with the first type of emission material between 3 and 6 min sputtering time, it can be assumed that the work function influenced mainly by the higher amount of the oxidized tungsten in case of the $\mathrm{Ba}-\mathrm{Y}$ emission material (15\% at Ba-Ca emission material and 25-30\% at $\mathrm{Ba}-\mathrm{Y}$ emission material). Up to $12 \mathrm{~min}$ ion bombardment, the amount of oxidized $\mathrm{W}$ decreased and reached a constant value by $15 \mathrm{~min}$. After $15 \mathrm{~min}$ of sputtering time the Ba amount was below the detection limit and the oxygen as well as the elemental tungsten had a constant value. The work function had an increasing trend until $24 \mathrm{~min}$ sputtering, similarly to the previous result.

By this time the surface corresponds to a Ba-free and slightly oxidized tungsten foil, and the measured work function value $(\Phi=4,7 \mathrm{eV})$ is in a good agreement with the value known from the literature.

The observed results led us to the conclusion that the following layer structure evolves on the $\mathrm{W}$ surface as the result of the sintering (reaction with Ba-containing emission materials at $1650^{\circ} \mathrm{C}$ in Ar atmosphere (Fig. 3). After sintering, the surface of the samples is covered with adsorbed $\mathrm{O}$ and $\mathrm{C}$ that can be easily removed by short intensive sputtering. This coverage is due to the adsorption of the contaminations inside the chamber. Below this layer the $\mathrm{BaO}$ coat is located. Using XPS and WFS methods, the experiment established that the $\mathrm{W}$ layer is more in the oxidized state than in case of $\mathrm{Ba}-\mathrm{Y}$ emission material under the $\mathrm{BaO}$ layer. Since both doublets of $\mathrm{W} 4 \mathrm{f}$ could be observed, it can be assumed that the elemental and oxidized tungsten are mixed in this layer. The bottom layer, the elemental tungsten was not reached during our investigation.

With the XPS MultiQuant Program [20] we also had the opportunity to calculate the $\mathrm{BaO}$ coverage that takes place during sintering. With the assumption of the purposed layer structure and using the measured concentration ratios, the $\mathrm{BaO}$ coverage is less than a monolayer $(\sim 60 \%)$. This led us to the conclusion that in the early life of the cathodes the coverage is not homogenous and we found disagreement with Rittner and Jensen's results [4,21].

In the next part of the investigation we heated the samples up to $700^{\circ} \mathrm{C}$ under UHV conditions. In Fig. 4 the XPS concentra- 
tion ratio and work function values are shown as a function of temperature in case of $\mathrm{Ba}-\mathrm{Ca}$-containing emission material.

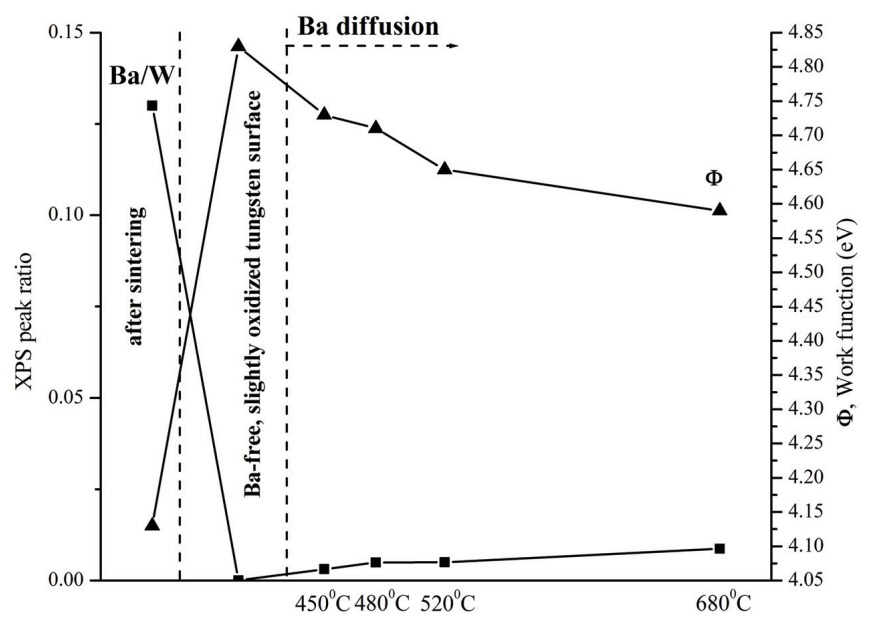

Fig. 4. The temperature dependence of $\mathrm{Ba}$ in case of $\mathrm{Ba}-\mathrm{Ca}$-containing emission material. The Ba diffusion could be detected at $450^{\circ} \mathrm{C}$

After the sintering process the work function is $4,1 \mathrm{eV}$ and the $\mathrm{Ba} / \mathrm{W}$ ratio is 0,13 . With extensive ion bombardment, we cleaned the surface of the $\mathrm{Ba}$ in order to achieve the slightly oxidized tungsten surface. For the further experiments, this surface was detected. During the heating process of this surface, the $\mathrm{Ba}$ diffused through the hole and it could be detected around $450^{\circ} \mathrm{C}$ resulting in a decreasing work function value. For further temperature increase, the $\mathrm{Ba} / \mathrm{W}$ ration continuously increased and, in a good agreement with it, the $\Phi$ decreased.

The same experiment was carried out in case of Ba-Y emission material; the $\mathrm{Ba}$ diffusion started at higher temperature, $600^{\circ} \mathrm{C}$. Despite of the minimum $\mathrm{Ba}$ amount on the surface ( $\mathrm{Ba} / \mathrm{W}$ concentration ratio is 0,0031 at $450^{\circ} \mathrm{C}$ for $\mathrm{Ba}-\mathrm{Ca}$ emission material and 0,0060 at $600^{\circ} \mathrm{C}$ for $\mathrm{Ba}-\mathrm{Y}$ emission material), we could observe the decreasing tendency for the work function.

The Ba diffusion results confirm the above statement, that the $\mathrm{W}$ layer is more in the oxidized state in case of $\mathrm{Ba}-\mathrm{Y}$ emission material: the $\mathrm{Ba}$ diffusion started at higher temperature at $\mathrm{Ba}-\mathrm{Y}$ emission material, leaving more time for oxidation of the tungsten foil.

\section{Conclusion}

In this paper the interaction between the emission material and tungsten surface was revealed, by carrying out experiments on tungsten foils. Our aim was to model a tungsten cathode tip surface during lamp operation. Due to the high operating temperature, the investigation of the cathode's surface has difficulties, but the goal could be achieved by examine tungsten surface under UHV conditions using XPS and WFS methods.

It was established experimentally that $\mathrm{BaO}$ evolves on the $\mathrm{W}$ surface after the high temperature sintering process. The XPS concentration results implied that the $\mathrm{BaO}$ coverage is less than a monolayer $(60 \%)$ at each sample. Temperature dependence of Ba diffusion was also established and compared at two emis- sion materials that are currently used in the industry. In case of the $\mathrm{Ba}-\mathrm{Ca}$ emission material, the $\mathrm{Ba}$ diffusion started at $450^{\circ} \mathrm{C}$, while in case of the $\mathrm{Ba}-\mathrm{Y}$ emission material at $600^{\circ} \mathrm{C}$ for.

The results of the model experiments led us to the conclusion that the cathodes have a physisorbed oxide layer on the surface, and the structure of the tips can be described as follows:

physisorbed oxide, carbon layer on / Ba-O (e.g. BaO) / (slightly) oxidized tungsten / elemental tungsten.

Using XPS and WFS methods, the experience established that the $\mathrm{W}$ layer is more in the oxidized state than in case of $\mathrm{Ba}-\mathrm{Ca}$ emission material under the $\mathrm{BaO}$ layer.

\section{References}

1 Krahl-Urban B, Niekisch EA, Wagner H, Work function of stepped tungsten single crystal surfaces, Surface Science 64 (1977), 52.

2 Riedel M, Düsterhöft H, Nágel F, Investigation of tungsten cathodes activated with Ba2CaWO6, Vacuum 61 (2001), 169.

3 Bhalla RS, Improved electron emission materials for HID lamps, Journal of the Illuminating Engineering Society (1979), 174.

4 Rittner ES, Alert RH, Rutledge W C, Studies on the Mechanism of Operation of the L Cathode, J. Appl. Phys. 28 (1957), 156.

5 Forman R, Surface studies of barium and barium oxide on tungsten and its application to understanding the mechanism of operation of an impregnated tungsten cathode, J. Appl. Phys. 47 (1976), no. 12, 5272.

6 Haas GA, Marrian CRK, Shih A, Interpretation of AES data of impregnated cathodes, Appl. Surf. Sci. 24 (1985), 430.

7 Bailey P, Cowie, D. Norman BCC, Operation and oxidation of thermionic dispenser cathodes studied by high resolution photoemission, J. Vac. Sci. Technol. A 16 (1998), no. 4, 2309.

8 Rittner ES, On the mechanism of operation of the type B impregnated cathode, J. Appl. Phys. 48 (1977), no. 10, 4344.

9 Springer RW, Haas TW, Auger electron spectroscopy study of cathode surfaces during activation and poisoning. I. The barium-on-oxygen-on-tungsten dispenser cathode, J. Appl. Phys. 45 (1974), 5260.

10 Jones D, McNeely D, Swanson LW, Surface and emission characterization of the impregnated dispenser cathode, Appl. Surf. Sci. 2 (1979), 232.

11 Forman $\mathbf{R}$, The role of oxygen in the low work function surface complex of barium on oxygen on tungsten (substrate), Appl. Surf. Sci. 25 (1986), 13.

12 Ravi M, Bhat KS, Estimation of barium evaporation rate from emission measurement of dispenser cathodes, Appl. Surf. Sci. 215 (2003), 33.

13 Kudo M, Sakai Y, Ichinokawa T, Dependencies of secondary electron yields on work function for metals by electron and ion bombardment, Applied Physics Letter 76 (2000), no. 23, 3475.

14 Vida Gy, Josepovits VK, Győr M, Deák P, Characterization of Tungsten Surfaces by Simultaneous Work Function and Secondary Electron Emission Measurements, Microsc. Microanal. 9 (2003), 337.

15 Scholtes J, Applications of work function microscopy in the onset mode, Fresenius' Journal of Analytical Chemistry 353 (1995), 499.

16 Verhoeven JAT, van Doveren H, XPS studies on $\mathrm{Ba}, \mathrm{BaO}$ and the oxidation of Ba, Appl. Surf. Sci. 5 (1980), 361.

17 Musket RG, Room-temperature adsorption of oxygen on tungsten surfaces: A review, J- Less-Common metals 22 (1979), 175.

18 Ptushinkii Yu G, Chuikov BA, Soviet Phys.-Solid State 10 (1968), 565.

19 Hopkins BJ, Pender KR, The adsorption of oxygen on the surfaces of (110) and (100) oriented tungsten single crystals, Surface Sci. 5 (1966), 155.

20 Mohai M, Surf. Interface Anal. 36 (2004), 828.

21 Jensen KL, Lau YY, Levush B, XPS MultiQuant for Windows, 1999-2005, IEEE Transactions on Plasma Science 28 (2000), no. 3, 772. 